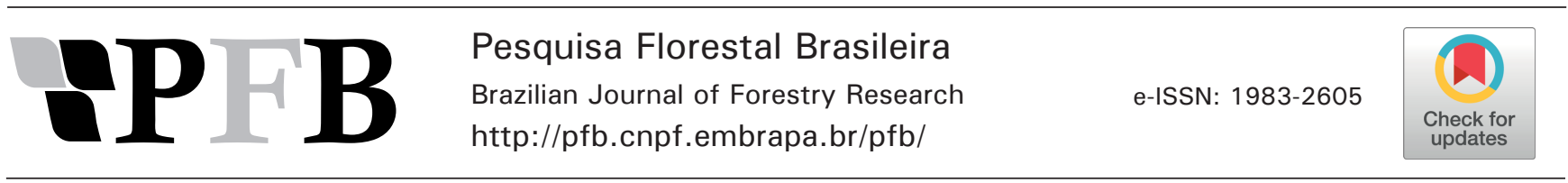

\title{
Estimativa do material combustível em área de Cerrado campo sujo a partir de imagens do sensor RGB
}

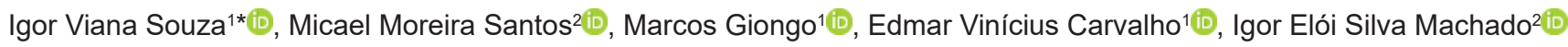 \\ ${ }^{1}$ Universidade Federal do Tocantins, Rua Badejós, Chácaras 69 e 72, Lt. 07, CEP 77402-970, Gurupi, TO, Brasil \\ ${ }^{2}$ Universidade Federal do Paraná, Av. Prefeito Lothário Meissner, 632, Jardim Botânico, CEP 80210 -170, Curitiba, PR, Brasil
}

"Autor correspondente:

igorvianasousa@gmail.com

Termos para indexação:

Sensoriamento remoto

Modelos digitais

Biocarburante

Index terms:

Biofuels

Digital models

Remote sensing

Histórico do artigo:

Recebido em 17/08/2018

Aceito em 12/12/2018

Publicado em 29/12/2018

doi: 10.4336/2018.pfb.38e201801706
Resumo - A quantificação do material combustível em área do Cerrado campo sujo é limitada pela dificuldade em obtenção de dados, nos altos custos e no elevado tempo gasto em campo. Em busca de alternativas que facilitem a obtenção dos dados, o uso de sensores RGB se destaca, podendo ser uma ferramenta útil e eficaz na quantificação do material combustível. Nesse contexto, o trabalho teve como objetivo avaliar a viabilidade da utilização de imagens provenientes de um sensor RGB aerotransportado por um multirotor para estimava do material combustível por meio da análise de regressão. Foi realizada a amostragem do material combustível da área que foi pesada em campo e seca em estufa. Com o processamento das imagens digitais foram obtidas a altura ( $\mathrm{ht}_{\mathrm{MDA}}$ ) e o índice de vegetação (NGRDI) dos pixels que abrangiam as unidades amostrais, seguidos de análise de correlação entre dados do processamento digital e o material combustível. Posteriormente, foram ajustados três modelos de regressão, em que foram obtidos coeficiente de determinação ajustados $\left(\mathrm{R}^{2}{ }_{\text {aj }}\right)$ de 0,39 a 0,80 . O uso dos sensores RGB apresentam potencial para a estimação de material combustível. Quando se combina as variáveis ht ${ }_{\mathrm{MDA}}$ e NGRDI, são obtidos valores mais próximos da linha média de distribuição.

\section{Estimation of combustible material in Cerrado grassland area from RGB sensor images}

\begin{abstract}
The quantification of fuel material in the Cerrado area is limited by the difficulty in obtaining data, the high costs and the high time spent in the field. In search of alternatives that facilitate the data acquisition, the use of RGB sensors stands out being able to be a useful and effective tool in quantifying the combustible material. In this context, the objective of this work was to evaluate the feasibility of using images from an airborne RGB sensor by a multirotor to estimate the combustible material by means of regression analysis. The fuel material was sampled from the area that was weighed in the field and dried in an oven. With the digital images processing, the height (htMDA) and the vegetation index (NGRDI) of the pixels covering the sample units were obtained, followed by a correlation analysis between the digital processing data and the combustible material. Subsequently, three regression models were adjusted, in which adjusted coefficient of determination $\left(\mathrm{R}^{2} \mathrm{aj}\right)$ was obtained from 0.39 to 0.80 . The use of RGB sensors has potential for estimation of combustible material. When the htMDA and NGRDI variables are combined, values closer to the mid-range are obtained.
\end{abstract}




\section{Introdução}

Novas técnicas para a quantificação de material combustível em área do Cerrado têm sido estudadas, com o intuito de buscar alternativas que facilitem a obtenção dos dados (Tavares, 2017). De acordo com Batista et al. (2017), o material combustível é qualquer material orgânico suscetível que participa no processo de combustão, sendo que sem o seu controle podem ocorrer em incêndios desastrosos. Dentre as técnicas para quantificação do material combustível, destacase a coleta do material vegetal superficial, por meio de amostras destrutivas em campo, considerado o método mais preciso (Tavares, 2017). No entanto, com a heterogeneidade e diversidade do bioma Cerrado, a quantificação por meio da estimativa direta é onerosa e complexa (Ferraz et al., 2014).

Em razão da complexidade em estimar o material combustível por meios diretos, os métodos indiretos destacam-se, pois estão baseados na correlação da biomassa com variáveis independentes e fáceis de serem mensuradas em campo, como por exemplo a altura, ou por sensoriamento remoto (Bendig et al., 2014).

A utilização de sensores RGB de alta resolução, aerotransportado por aeronaves remotamente pilotadas (RPA) para obtenção de estimativa da vegetação, torna o sensoriamento remoto um instrumento benéfico em pesquisas de campo e com resultados precisos e acurados (Fornace, 2014). Fato que justifica-se pela facilidade e quantidade de dados que os sensores remotos dispõem, tornando-os alternativas viáveis para estimativa da carga de material combustível.

Lati et al. (2013) relatam a potencialidade da fotogrametria, sendo possível estimar a biomassa da vegetação por meio da altura da vegetação, sendo esta uma variável com correlação forte e positiva e obtida com a modelagem da superfície após o processamento das imagens feitas por levantamento aéreo. Nesse ponto, trabalhos como os de Zhang et al. (2016), Cunliffe et al. (2016), e Bendig et al. (2014) são exemplos da utilização de aeronaves remotamente pilotadas como ferramenta para obter estimativas da vegetação utilizando a altura.

Para Jannoura et al. (2015), o processamento digital com imagens obtidas de sensores RGB, quando transformados em índices de vegetação tal como índice de diferença normalizada vermelho-verde (NGRDI), são ferramentas eficazes na estimava de parâmetros da vegetação, substituindo o índice de vegetação da diferença normalizada (NDVI) que requer sensor que alcance o espectro infravermelho. Em seus resultados, o sensor RGB mostrou-se uma ferramenta potencial para obter parâmetros da vegetação, como o acúmulo de biomassa.

Este trabalho teve por objetivo avaliar a viabilidade de imagens provenientes de um sensor RGB aerotransportado por um multirotor, para estimar o material combustível em uma área de pastagem em estado de senescência situada em área de campo sujo no Cerrado, por meio de análise de regressão com os dados de campo.

\section{Material e métodos}

O trabalho foi realizado em outubro de 2017, em local situado próximo ao Centro de Monitoramento Ambiental e Manejo do Fogo (CeMAF), no munícipio de Gurupi, na região Sul do Estado do Tocantins. Apresenta altitude média de $280 \mathrm{~m}$, sob coordenadas $11^{\circ} 44^{\prime} 8^{\prime \prime} \mathrm{S}$ e $49^{\circ} 02^{\prime} 58^{\prime \prime} \mathrm{W}$, onde se encontra uma área de pastagem em Cerrado campo sujo em processo de regeneração natural com 6,30 ha, com predominância de vegetação rasteira em estado de senescência e poucas espécies arbóreas. De acordo com a classificação do clima Thornthwaite, o tipo climático predominante na região é $\mathrm{C} 2 \mathrm{wA}$ " $a$ ", caracterizando-se como clima subúmido, com moderada deficiência hídrica no inverno, com evapotranspiração potencial média anual de $1.483 \mathrm{~mm}$ e temperatura média de $26,6{ }^{\circ} \mathrm{C}$ (Tocantins, 2015).

\section{Amostragem da biomassa}

Foram delimitadas 38 unidades amostrais de $1 \mathrm{~m}^{2}$ (1 $\mathrm{m} \mathrm{x} 1 \mathrm{~m})$, distribuídas de forma aleatória, visando melhor representatividade e controle da mensuração da área (Figura 1).

A coleta de todo material suscetível ao processo de combustão presente em cada unidade amostral foi realizada no mesmo dia e após o voo do multirotor.

O material foi pesado em campo para medição do material combustível, sendo separadas subamostras que foram levadas para secagem a $70^{\circ} \mathrm{C}$ durante $120 \mathrm{~h}$ em estufa. Após, foram pesadas novamente para obter o peso seco e dessa forma estimar a massa do material combustível (MC) em cada unidade amostral, em $\mathrm{kg} \mathrm{m}^{-2}$. 


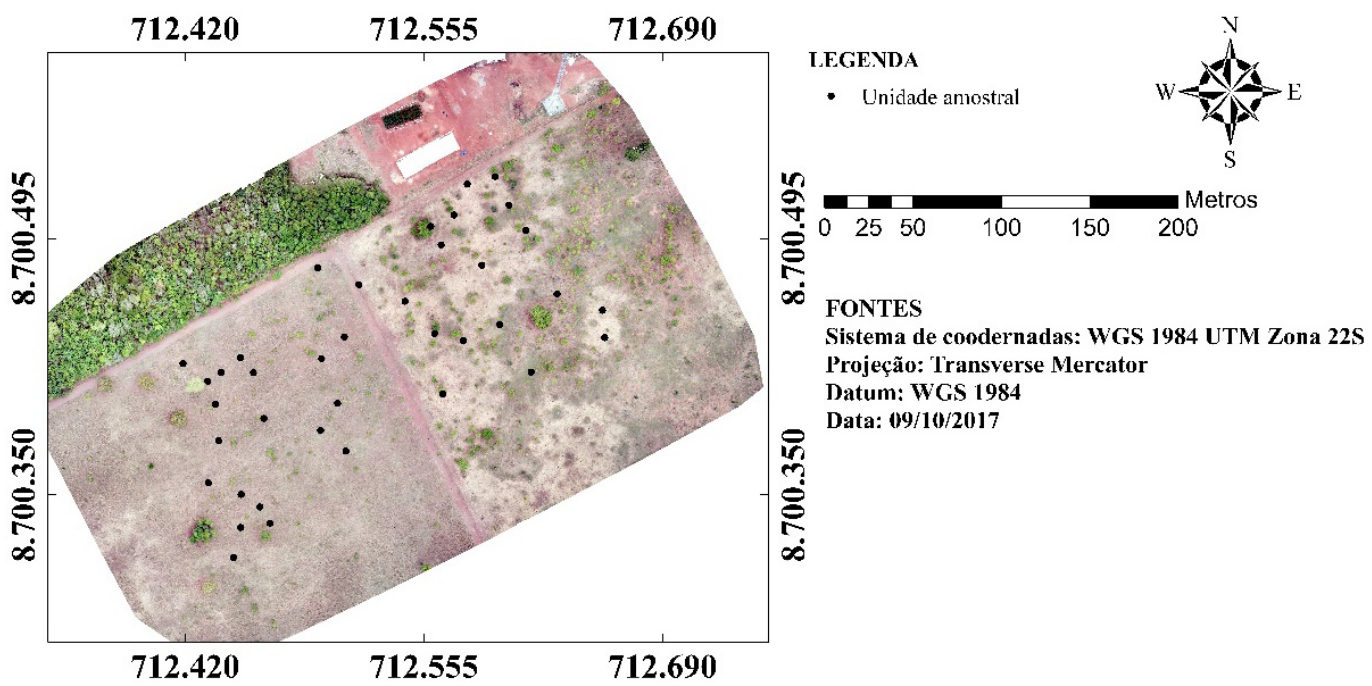

Figura 1. Distribuição das unidades amostrais para estimativa de biomassa, em Cerrado campo sujo, em Gurupi, TO.

Figure 1. Distribution of sample units to estimate biomass in Cerrado grassland, Gurupi, Tocantins State.

\section{Levantamento aéreo fotogramétrico}

Foi utilizado um multirotor com sensor RGB nas dimensões de $1 / 2.3$ " com resolução 4.000 x 3.000 pixels e distância focal de $4,73 \mathrm{~mm}$. Foi definida a altura do voo de $80 \mathrm{~m}$ e um recobrimento de $75 \%$ longitudinal e $75 \%$ lateral que resultou em um ground sample distance (GSD) de 2,50 cm, valor de representação do pixel da imagem em unidades do terreno. No levantamento da área foram registradas 242 fotos em uma área de 6,30 ha em $10 \mathrm{~min}$. Foram distribuídos uniformemente dez pontos de controle e 38 marcações nas unidades amostrais, tornando-as facilmente identificáveis nas imagens. Em seguida foram realizadas mensurações dos pontos com GPS geodésico, com objetivo de serem usados como pontos de controle (ground control points $G C P s$ ) no processamento das imagens, proporcionando o georreferenciamento das imagens em relação ao solo com maior acurácia.

\section{Geração do modelo digital de altura normalizada}

Nesta fase, foi utilizado o algoritmo structure from motion (SfM) que é um método fotogramétrico para criar modelos tridimensionais a partir de várias fotografias sobrepostas para a reconstrução da cena fotografada (Verhoeven, 2011).

A reconstrução da geometria em $3 \mathrm{D}$ permite a exportação do modelo digital de superfície (MDS) e modelo digital de terreno (MDT) para geração do modelo digital de altura normalizada, subtraindo-se o MDT do MDS. Posteriormente, obteve-se a altura média de cada unidade amostral $\left(\mathrm{ht}_{\mathrm{MDA}}\right)$.

\section{Índice de diferença normalizada vermelho-verde (NGRDI)}

As imagens foram combinadas aplicando-se o algoritmo NGRDI em que é realizada a calibração radiométrica e posteriormente o cálculo utilizando as variáveis band green e band red (equação 1).

$$
\mathrm{NGRDI}=\frac{\text { greenDN }- \text { redDN }}{\text { greenDN }+ \text { redDN }}
$$

Em que: GreenDN é a região do espectral do verde normalizada e $R e d \mathrm{DN}$ é a região espectral vermelha normalizada.

Os dados do índice de vegetação foram calculados e extraídos com auxílio software SIG para cada unidade amostral.

Posteriormente, foi realizada a análise descritiva da altura do modelo digital $\left(\mathrm{ht}_{\mathrm{MDA}}\right)$, do índice de diferença normalizada vermelho-verde (NGRDI) e do material combustível (MC). A média do índice de vegetação (NGRDI) foi de -0,1729, com desvio padrão aproximado de 0,0109. A altura média do modelo digital foi cerca de $21,14 \mathrm{~cm}$ com desvio padrão de $19,54 \mathrm{~cm}$. Com relação ao material combustível, foi obtido valor de $0,3434 \mathrm{~kg} \mathrm{~m}^{-2}$ com desvio padrão de $0,1628 \mathrm{~kg} \mathrm{~m}^{-2}$. 


\section{Análise estatística}

A análise estatística de correlação (Pearson, $\mathrm{p}<0,05$ ) foi realizada entre as variáveis $\mathrm{ht}_{\mathrm{MDA}}(\mathrm{cm})$, NGRDI e $\mathrm{MC}\left(\mathrm{kg} . \mathrm{m}^{-2}\right)$ e suas respectivas transformações como variáveis dos modelos digitais, sendo elas: $\mathrm{NGRDI}^{2}$, $\mathrm{NGRDI}^{3}, \mathrm{ht}^{2}{ }_{\mathrm{MDA}}, \mathrm{ht}^{3}{ }_{\mathrm{MDA}}$, Log ht ${ }_{\mathrm{MDA}}$ e Raiz ht ${ }_{\mathrm{MDA}}$, visando ampliar a quantidade de variáveis independentes a serem utilizadas nas análises.

Para análise de regressão (Método Backward) foi considerado como variável dependente o material combustível e variáveis independente $\mathrm{ht}_{\mathrm{MDA}}(\mathrm{cm})$, NGRDI e suas respectivas transformações. Foi utilizado o modelo matemático citado por Canavesi et al. (2010) (equação 2).

$$
Y=\beta 0+\beta 1 \chi 1+\beta 2 \chi 2+\beta 3 \chi 3+\ldots \beta N \chi N+\varepsilon
$$

Em que: $Y=$ variável dependente, $\chi=$ variáveis independentes e $\beta=$ coeficientes gerados das seleções das variáveis.

Os melhores modelos foram escolhidos por meio da análise gráfica dos resíduos padronizados, erro padrão da estimativa em porcentagem (Syx\%) e valores do coeficiente de determinação ajustado $\left(\mathrm{R}^{2}{ }_{\text {aj }}\right)$.

\section{Resultados}

Na Tabela 1 são apresentadas as análises de correlação de Pearson entre as variáveis altura total $\left(\mathrm{ht}_{\mathrm{MDA}}\right)$ e material combustível (MC), índice de diferença normalizada vermelho-verde (NGRDI) e MC, e suas respectivas transformações como variáveis independentes.

Os coeficientes de correlação para NGRDI e MC; $\mathrm{NGRDI}^{2}$ e MC; $\mathrm{NGRDI}^{3}$ e $\mathrm{MC}$ foram consideradas de magnitude moderada e positiva, exceto para a variável $\mathrm{NGRDI}^{2}$, onde foi apresentada uma relação inversa $(r=-0,41)$. Para ht $t_{\mathrm{MDA}}$ e MC obteve-se um $r=0,83$, classificado como forte positiva, segunda classificação de Pearson.

Na Figura 2 é demostrada graficamente a dispersão dos resíduos padronizados dos modelos. Nota-se que no modelo 3, com a combinação das duas variáveis, os valores se apresentam mais próximos da linha média de distribuição, confirmando que as combinações entre as variáveis explicam melhor a relação do real e do estimado.

$\mathrm{Na}$ Tabela 2 estão apresentados os modelos de regressão ajustados e testados para a variável dependente, a partir dos dados da altura ( $\left.\mathrm{ht}_{\mathrm{MDA}}\right)$ e índices de vegetação (NGRDI).
Tabela 1. Coeficientes de correlação de Pearson entre o material combustível e a variável obtida do modelo digital de altura $\left(\mathrm{Ht}_{\mathrm{MDA}}\right)$ e índice de vegetação (NGRDI).

Table 1. Pearson correlation coefficients between the combustible material and the variable obtained from the digital height model (HtMDA) and vegetation index (NGRDI).

\begin{tabular}{cc}
\hline Variável & $\mathbf{r}$ \\
\hline $\mathrm{NGRDI}$ & $0,40^{*}$ \\
$\mathrm{NGRDI}^{2}$ & $-0,41^{* *}$ \\
$\mathrm{NGRDI}^{3}$ & $0,47 * *$ \\
$\mathrm{ht}_{\mathrm{MDA}}(\mathrm{cm})$ & $0,83 * *$ \\
$\mathrm{ht}_{\mathrm{MDA}}(\mathrm{cm})^{2}$ & $0,77 * *$ \\
$\mathrm{ht}_{\mathrm{MDA}}(\mathrm{cm})^{3}$ & $0,70^{* *}$ \\
$\mathrm{Log} \mathrm{ht}_{\mathrm{MDA}}(\mathrm{cm})$ & $0,79 * *$ \\
$\mathrm{Raiz} \mathrm{ht}_{\mathrm{MDA}}(\mathrm{cm})$ & $0,83 * *$ \\
\hline
\end{tabular}

Em que: NGRDI =índice de vegetação das unidades amostrais, $\mathrm{NGRDI}^{2} \mathrm{e}$ $\mathrm{NGRDI}^{3}=$ índice de vegetação das unidades amostrais ao quadrado e ao cubo, respectivamente; $\mathrm{ht}_{\mathrm{MDA}}(\mathrm{cm})$, altura $\mathrm{em} \mathrm{cm}$, extraída do modelo digital de altura das unidades amostrais; $\mathrm{Ht}^{2}{ }_{\mathrm{MDA}}(\mathrm{cm}) \mathrm{e} \mathrm{ht}^{3}{ }_{\mathrm{MDA}}(\mathrm{cm})$, altura $\mathrm{em} \mathrm{cm}^{2}$ e $\mathrm{cm}^{3}$, respectivamente, $\log =$ logaritmo na base $10 ;$ Raiz $=$ raiz quadrada; $\mathrm{r}=$ correlação de Pearson; * e ** = significativo a $5 \%$ e $1 \%$ pelo teste $\mathrm{T}$, respectivamente.

Tabela 2. Modelos de regressão lineares testados e ajustados para estimar o material combustível, em Cerrado campo sujo, em Gurupi, TO.

Table 2. Linear regression models tested and adjusted to estimate the fuel material in Cerrado grassland, Gurupi, Tocantins State.

\begin{tabular}{|c|c|c|c|c|}
\hline $\mathbf{N}$ & Modelo de regressão & $\mathbf{R}^{2}{ }_{\mathrm{AJ}}$ & Syx & Syx $\%$ \\
\hline 1 & $\mathrm{MC}=(0,198+0,1 \mathrm{HtMDA} *(0,0007)+\varepsilon$ & 0,68 & 0,09 & 26,76 \\
\hline 2 & $\begin{array}{l}\mathrm{MC}=-4,562+\mathrm{NGRDI} *(48 \mathrm{M} 437+ \\
\mathrm{NGRDI}^{3}(663,240)+\varepsilon\end{array}$ & 0,39 & 0,12 & 36,72 \\
\hline 3 & $\begin{array}{l}\text { MC }=-3,72+\text { HTmda } *(0.018)+\text { htMDA } \\
*(0,00012)+\text { LOG }(\text { htMDA }) *(0,235)+ \\
\text { NGRDI* }(-38,789)+\text { NGRDI }^{3} *(509,233) \\
+\varepsilon\end{array}$ & 0,80 & 0,71 & 20,56 \\
\hline
\end{tabular}

Em que: $\mathrm{N}=$ número do modelo; $\mathrm{MC}=$ material combustível $\left(\mathrm{kg} \cdot \mathrm{m}^{-2}\right) ; \mathrm{R}^{2} \mathrm{aj}$ $=$ coeficiente de determinação ajustado; Syx = erro padrão da estimativa; Syx $(\%)=$ erro padrão da estimativa em porcentagem; $\mathrm{e}+\varepsilon=$ resíduos.

Destaca-se na Figura 2 o modelo que apresentou o melhor ajuste.

Assim, foram obtidas equações para variável dependente, sendo o modelo 1 ajustado para a variável independente $\mathrm{ht}_{\mathrm{MDA}}$, modelo 2 ajustado para NGRDI e modelo 3 ajustado para ht ${ }_{\mathrm{MDA}}$ e NGRDI. 


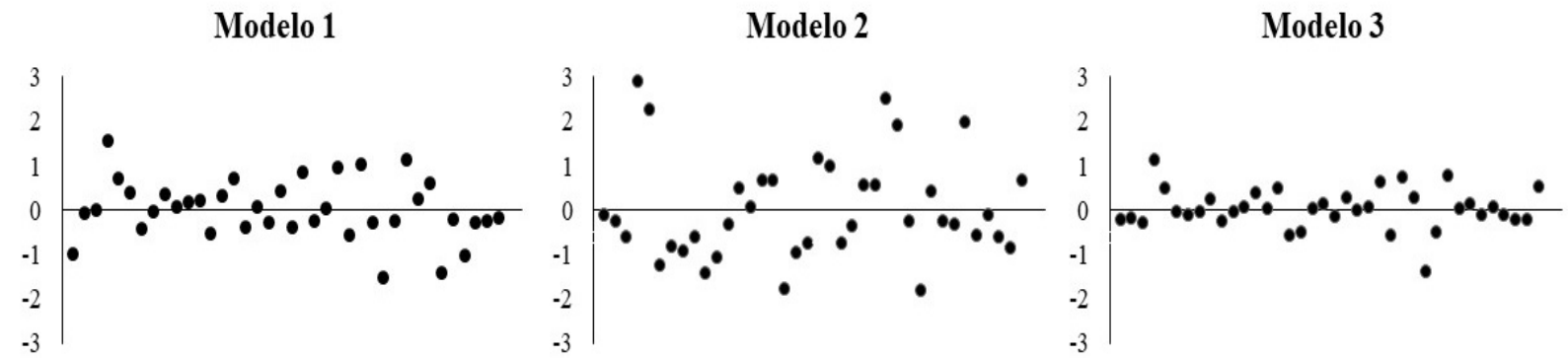

Figura 2. Distribuição dos resíduos padronizados dos modelos testados e ajustados para estimar o material combustível, em Cerrado campo sujo, em Gurupi, TO.

Figure 2. Distribution of residuos standardized model tested and adjusted to estimate the fuel material in Cerrado grassland, Gurupi, Tocantins State

\section{Discussão}

O valor médio do índice de diferença normalizada vermelho-verde (NGRDI) encontrado neste trabalho $(-0,1729)$ é um indicativo do processo de senescência da vegetação herbácea, resultado que pode ser observado em campo. Segundo Hunt et al. (2005), valores próximos a zero do índice de vegetação é consequência do baixo teor de água e clorofila contidas nas estruturas da planta, ocasionando o aumento da reflectância no espectro visível, valores que refletem o cálculo do NGRDI.

Bendig et al. (2014), em sua pesquisa em um campo experimental de cevada, encontraram correlação de 0,82 entre a variável (altura), obtida no modelo digital, e biomassa. Apesar dos autores trabalharem com agricultura de precisão, o valor assemelha-se com o obtido neste trabalho, resultado que mostra forte correlação entre a variável altura e a biomassa.

Para as variáveis NGRDI e material combustível (MC) observou-se correlação significativa de $r=0,47$, no entanto, essa correlação não foi tão forte quanto as relatadas por Jannoura et al. (2015) e Hunt et al. (2005), que obtiveram correlações variando de 0,55 a 0,74 e 0,63 a 0,94, respectivamente, para cultura de ervilhas. Tal diferença pode ser explicada pelo tipo de vegetação estudada que difere do campo Cerrado estudado neste trabalho, caracterizado por heterogeneidade da vegetação.

\section{Análise dos modelos ajustados}

$\mathrm{O}$ valor $\mathrm{R}^{2}$ ajustado para a altura total ( $\mathrm{ht}_{\mathrm{MDA}}$ ) foi de 0,68 , sendo próximo a obtidos em outras pesquisas semelhantes. Por exemplo, Bending et al. (2014) trabalharam somente com estimativa de biomassa por meio da altura obtidas no modelo digital e obtiveram valores que variaram entre 0,39 a 0,68 , para cultura de cevada utilizando modelo exponencial. Tumlisan (2017) utilizando o mesmo método para estimar biomassa, encontrou um $\mathrm{R}^{2}$ ajustado próximo a 0,71 para cultura de milho. Segundo Palhares et al. (2008), as herbáceas são consideradas plantas de excelente taxa fotossintética, sendo possível perceber a estreita relação entre o índice de área foliar e altura da vegetação, tornando a premissa adequada para estimar a biomassa por meio da altura.

Tumlisan (2017) utilizou índices de vegetação (excess green minus red) baseados em imagens RGB com o mesmo objetivo de predizer a biomassa seca, obtendo $\mathrm{R}^{2}$ ajustados próximos de 0,41 , sendo este valor semelhante aos obtidos neste trabalho $(0,39)$, utilizando a variável independente NGRDI.

A combinação entre as variáveis independente com suas transformações, $\mathrm{ht}_{\mathrm{MDA}}$ e NGRDI resultou em um $\mathrm{R}^{2}$ ajustado maior $\left(\mathrm{R}_{\mathrm{aj}}^{2}=0,80\right)$ e a distribuição de resíduos padronizado mais próximo da linha média de distribuição comparado aos modelos 1 e 2, quando utilizou-se somente uma variável independente para estimar o material combustível (Tabela 2). Tumlisan (2017) obteve melhor resultando quando combinou no modelo a altura do modelo digital e índices de vegetação, e destacou a importância de se utilizar mais variáveis para estimar a biomassa da cultura, para se obter resultados próximos da realidade. Ainda, Geipel et al. (2014) e Bendig et al. (2015) ressaltam que a combinação de variáveis permite estimativas avançadas, melhorando significativamente o desempenho do modelo, tendo ganhos nos ajustes e diminuição do erro padrão residual. 


\section{Conclusões}

A estimativa do material combustível analisadas por regressão, utilizando imagens provenientes de um sensor RGB aerotransportado por um multirotor mostrou-se eficiente.

A altura do modelo digital ( $\left.\mathrm{ht}_{\mathrm{MDA}}\right)$ é uma variável com potencial para a estimação do material combustível.

O modelo composto pela combinação entre as variáveis independentes $\mathrm{ht}_{\mathrm{MDA}}$ e índice de diferença normalizada vermelho-verde (NGRDI), apresentaram os melhores resultados para estimativa da biomassa em Cerrado campo sujo.

\section{Referências}

Batista, A. C. et al. Incêndios florestais. Curitiba: UFPR, 2017. 47 p.

Bendig, J. et al. Combining UAV-based plant height from crop sufarce models, visible and near infrared vegetation indices for biomass monitoring in barley. International Journal of Applied Earth Observation and Geoinformation, v. 39 p. 79-87, 2015. DOI: 10.1016/j.jag.2015.02.012.

Bendig, J. et al. Estimating biomass of barley using Crop Surface Models (CSMs) derived from UAV: based RGB imaging. Remote sensing, v. 6, n. 11, p. 10395-10412, 2014. DOI: 10.3390/rs61110395.

Canavesi, V. et al. Estimativa de volume de madeira em plantios de Eucalyptus spp. utilizando dados hiperespectrais e dados topográficos. Revista Árvore, v. 34, n. 3, p. 539-549, 2010.

Cunliffe, A. M. et al. Ultra-fine grain landscape-scale quantification of dryland vegetation structure with drone-acquired structure-frommotion photogrammetry. Remote Sensing of Environment, v. 183, p. 129-143, 2016. DOI: 10.1016/j.rse.2016.05.019.

Ferraz, A. S. et al. Estimativa do estoque de biomassa em um fragmento florestal usando imagens orbitais. Floresta e Ambiente, v. 21, n. 3, p. 286-296, 2014.
Fornace, K. M. et al. Mapping infection disease landscapes: unmanned aerial vehicles and epidemiology. Trend in Parisitology, v. 30, n. 11, 2014.

Geipel, J. et al. Combined spectral and spatial modeling of corn yield based on aerial imagens and crop sufarce models acquide with an unmanned aircraft system. Remote Sensing, v. 6, n. 11, p. 1033510355, 2014. DOI: 10.3390/rs61110335.

Hunt, E. R. et al. Evaluation of digital photography from model aircraft for remote sensing of crop biomass and nitrogen status. Precision Agriculture, v. 6, p. 359-378, 2005.

Jannoura, R. et al. Monitoring of crop biomass using true colour aerial photographs taken from a remote controlled hexacopter. Biosystems Engineering, v. 129, p. 341-351, 2015. DOI: 10.1016/j. biosystemseng.2014.11.007.

Palhares, D. et al. Respostas fotossintéticas de plantas de cerrado nas estações seca e chuvosa. Revista Brasileira de Biociências, v. 8, n. 2, p. 213-220, 2010.

Lati, R. N. et al. Estimating plant growth parameters using an energy minimization-based stereovision model. Computer and Electronics in Agriculture. v. 98, p. 260-271, 2013. DOI: 10.1016/j. compag.2013.07.012.

Tavares, M. E. F. Metodologias usadas na quantificação de material combustível no Cerrado. Vértices, v. 19, n. 1, p. 175-182, 2017.

Tocantins. Secretaria do Planejamento Superintendência do Planejamento e Gestão Central de Políticas Públicas. Diretoria de Zoneamento Ecológico-Econômico. Base de dados geográficos do Tocantins. Palmas, 2015.

Tumlisan, G. Y. G. Monitoring growth develoment and yield estimation of maize using very high-resolution uav-images in Gronau, Germany. 2017. 52 f. Dissertação (Mestrado em Geo - Ciência da Informação e Observação da Terra) - University of Twent, Netherlands.

Verhoeven, G. Taking computer vision aloft: archaeological threedimensional reconstructions from aerial photographs with PhotoScan. Archaeological Prospection, v. 18, p. 67-73, 2011.

Zhang, J. et al. Seeing the forest from drones: testing the potential of lightweight drones as a tool for long-term forest monitoring. Biological Conservation, v. 198, p. 60-69, 2016. 